\title{
The Anti-Inflammation and Anti-Nociception Effect of Ketoprofen in Rats Could Be Strengthened Through Co-Delivery of a $\mathrm{H}_{2} \mathrm{~S}$ Donor, S-Propargyl- Cysteine
}

\author{
Yue $\mathrm{Yu}^{\mathrm{l}} *$ \\ Qinyan Yang ${ }^{1, *}$ \\ Zhou Wang' \\ Qian Ding' \\ Meng Li' \\ Yudong Fang' \\ Qida $\mathrm{He}^{\prime}$ \\ Yi Zhun Zhu (D) ${ }^{1,2}$
}

'State Key Laboratory of Quality Research in Chinese Medicine \& School of Pharmacy, Macau University of Science and Technology, Taipa, Macau SAR, People's Republic of China; ${ }^{2}$ Shanghai Key Laboratory of Bioactive Small Molecules \& School of Pharmacy, Fudan University, Shanghai, People's Republic of China

*These authors contributed equally to this work
Correspondence: Yi Zhun Zhu

Macau University of Science and Technology, Block E, Avenida Wai Long,

Taipa, Macau SAR, 999078, People's

Republic of China

Tel +85388972880

Fax +85328823575

Email yzzhu@must.edu.mo
Purpose: Ketoprofen (KETO) is a traditional non-steroidal anti-inflammatory drug (NSAIDs) with good analgesic and antipyretic effects. However, as NASIDs, the toxicity of KETO towards gastrointestinal (GI) system might limit its clinical use. S-propargylcysteine (SPRC) is an excellent endogenous $\mathrm{H}_{2} \mathrm{~S}$ donor showed wide application in the field of anti-inflammation, anti-oxidative stress, or even the protection of cardiovascular system through the elevation of endogenous $\mathrm{H}_{2} \mathrm{~S}$ concentration. As recently studies reported, co-administration of $\mathrm{H}_{2} \mathrm{~S}$ donor might potentially mitigate the GI toxicity and relevant side effects induced by series of NSAIDs.

Methods: In this study, we established a SPRC and KETO co-encapsulated poly (lactic-coglycolic acid) microsphere (SK@MS), and its particle size, morphology, storage stability and in vitro release profile were firstly investigated. The elevation of endogenous $\mathrm{H}_{2} \mathrm{~S}$ level of SK@MS was then calculated, and the pharmacodynamic study (anti-inflammation and analgesic effects) of SK@MS, SPRC, and KETO towards adjuvant induced arthritis (AIA) in rats were also studied. Finally, to test the potential side effect, the heart, liver, spleen, lung, kidney, stomach, small intestine, and large intestine were resected from rats and examined by H\&E staining.

Results: A monodispersed SK@MS could be observed under the SEM, and particle size was calculated around $25.12 \mu \mathrm{m}$. The loading efficiency (LE) for SPRC and KETO were $6.67 \%$ and $2.64 \%$, respectively, while the encapsulation efficiency (EE) for SPRC and KETO were $37.20 \%$ and $68.28 \%$, respectively. SK@MS showed a sustained release of SPRC and KETO in vitro, which was up-to 15 days. SK@MS could achieve a long-term elevation of the $\mathrm{H}_{2}$ $\mathrm{S}$ concentration in vivo, while SPRC showed an instant $\mathrm{H}_{2} \mathrm{~S}$ elevation and metabolize within $6 \mathrm{~h}$. Interestingly, the KETO did not show any influence on the $\mathrm{H}_{2} \mathrm{~S}$ concentration in vivo. After establishment of AIA model, neither SPRC nor KETO showed scarcely antiinflammation and anti-nociception effect, while conversely, SK@MS showed an obvious mitigation towards paw edema and pain in AIA rats, which indicated an improved antiinflammation and anti-nociception effect when co-delivery of SRC and KETO. Besides, low stimulation towards major organs in rats observed in any experimental group.

Conclusion: A monodispersed was successfully prepared in this study, and SK@MS showed a sustained SPRC and KETO release in vitro and $\mathrm{H}_{2} \mathrm{~S}$ release in vivo. In the pharmacodynamics study, SK@MS not only exhibited an excellent anti-inflammation and analgesic effects in AIA rats but also showed low stimulation towards rats.

Keywords: $\mathrm{H}_{2} \mathrm{~S}$ donor, ketoprofen, adjuvant induced arthritis, anti-inflammation, analgesic effect 


\section{Introduction}

Ketoprofen (KETO) is a non-steroidal anti-inflammatory drug (NSAIDs) with good antipyretic, analgesic, and antiinflammatory effects. ${ }^{1-6}$ Compared with other NSAIDs, KETO has obvious analgesia for inflammatory pain and postoperative pain and has less side effects. ${ }^{7,8}$ There have also been related studies in clinical medicine in recent years. $^{2,9,10}$ Ketoprofen and its related preparations are widely used in the treatment of inflammation (arthritis, scissor synovitis, ankylosing spondylitis, etc.), ${ }^{11,12}$ trauma $^{13,14}$ and postoperative pain. ${ }^{7,15}$ However, as a kind of NSAIDs, its side effects, such as gastrointestinal toxicity, ${ }^{16,17}$ are unavoidable, which potentially limits the clinical application of NSAIDs.

Hydrogen sulfide $\left(\mathrm{H}_{2} \mathrm{~S}\right)$, with a smell of rotten eggs, has long been regarded as toxic gas. ${ }^{18}$ However, recently, studies have gradually revealed its potential use as endogenous gasotransmitter. ${ }^{19-22}$ S-propargyl-cysteine (SPRC) is an analogue of garlic extract which could produce endogenous $\mathrm{H}_{2} \mathrm{~S}$ in vivo through upregulation of the expression of the cystathionine- $\gamma$-lyase (CSE) ${ }^{23}$ In recent years, studies have found that SPRC plays an important role in cardiovascular protection, ${ }^{24}$ anti-oxidative stress, ${ }^{25}$ and anti-inflammatory. ${ }^{23}$ All the therapeutical effects of SPRC were all reported to be relevant to the promotion of endogenous $\mathrm{H}_{2} \mathrm{~S}$ from SPRC.

Recently, studies have reported that covalently linkage of $\mathrm{H}_{2} \mathrm{~S}$-releasing donor together with NSAIDs could dramatically reduce the gastrointestinal toxicity and increase the therapeutical potency. ${ }^{26}$ Among which, ATB-352, a combination of KETO and $\mathrm{H}_{2} \mathrm{~S}$-releasing moiety, has been widely investigated for its high therapeutical effect and low side effect, which was proved possibly due to $\mathrm{H}_{2}$ S-releasing. ${ }^{27}$ A study led by Costa et al revealed that a marked enhancement of the potency and effectiveness towards pain of postoperative setting of ATB-352 could be observed when compared with the KETO. ${ }^{26}$ Whatsmore, the anti-inflammatory effect of ATB-352 on lipopolysaccharide-induced periodontitis in rats was also reported. ${ }^{28}$ However, to the best of our knowledge, whether codelivery of NSAIDs and $\mathrm{H}_{2} \mathrm{~S}$ donor could obtain a better analgesic effect and reduced gastrointestinal toxicity remains unclear.

Normally, the combination of two drugs has been considered to be a promising strategy to overcome toxicity and side effects when solely use one ingredient. ${ }^{29-33}$ As reported, many efforts have been made on a designing single carrier for two or more therapeutic agents, ${ }^{34-37}$ among which, the poly lactic-co-glycolic acid (PLGA) has been widely investigated for its high biocompatibility. ${ }^{38-42}$ Due to its compartmentalized internal structure, a water-in-oil-in-water (W1/O/W2) double emulsion method was normally used for the encapsulation of two cargos with different properties. ${ }^{43,44}$

In this study, SPRC and KETO were co-encapsulated in a PLGA microsphere (SK@MS) through the W1/O/W2 double emulsion method. The SK@MS showed a spherical-like structure, and monodispersed particle size. Besides, the sustained release up-to 15 days of SPRC and KETO from SK@MS could be observed. In the aspect of pharmacodynamics, the SK@MS showed a better anti-inflammation and anti-nociception effect in AIA rats when compared with solely use of SPRC or KETO.

\section{Materials and Methods \\ Materials}

S-Propargyl-Cysteine (SPRC) was synthesized as reported before. ${ }^{45,46}$ Poly lactic-co-glycolic acid (PLGA, 75:25, acid terminated, $15 \mathrm{k}$ ) was purchased from Evonik Industries AG (Essen, Germany). Polyvinyl alcohol (PVA) (87-89\% hydrolyzed, 13k) was obtained from Sigma-Aldrich (St. Louis, USA). Ketoprofen was purchased from Energy Chemical (Shanghai, China). Elisa kit of TNF- $\alpha$, IL-1 $\beta$, IL-6 and IL-10 were purchased from MultiSciences (Hangzhou, China). Phosphatebuffered saline (PBS), complete Freund's adjuvant (CFA), methanol, monobromobimane (MBB), hydrocortisone, diethylenetriaminepentaacetic acid (DTPA), dichloromethane (DCM), and acetonitrile were purchased from Macklin Industrial Corporation (Shanghai, China).

\section{The Preparation of SK@MS}

SPRC and KETO co-loaded PLGA microspheres (SK@MS) were prepared via the double emulsion solvent evaporation method with little modification. ${ }^{47}$ Briefly, $200 \mathrm{mg}$ of SPRC was firstly dissolved in $2000 \mu \mathrm{L}$ of distilled water to form the inner water phase (W1). The oil phase (O) was prepared through dissolving $1000 \mathrm{mg}$ of PLGA and $45 \mathrm{mg}$ of KETO in $20 \mathrm{~mL}$ of DCM, and the W1 was then dropwise added to the O. Next, the mixture was emulsified with homogenizer (T18 digital ULTRATURRAX $^{\circledR}$, IKA, Germany) at $7000 \mathrm{rpm}$ for $2 \mathrm{~min}$ in an ice bath. The obtained primary emulsion (W1/O) was then 
added into $2500 \mathrm{~mL}$ of $0.5 \%(\mathrm{w} / \mathrm{v})$ PVA solution and further emulsified by a homogenizer (T18 digital ULTRATURRAX $^{\circledR}$, IKA, Germany) at $7000 \mathrm{rpm}$ for $4 \mathrm{~min}$ to prepare the $\mathrm{W} 1 / \mathrm{O} / \mathrm{W} 2$ emulsion, and this $\mathrm{W} 1 / \mathrm{O} / \mathrm{W} 2$ emulsion was hardened under magnetic stirring (400 rpm) for 5 h. The obtained particles were collected through centrifugation, washed 3 times with distilled water and lyophilized. Finally, the lyophilized particles within the range from 20 to 30 micron were selected through sieve and named as SK@MS.

\section{Characterization of SK@MS}

The Microtrac S3500 (Montgomeryville, USA) was chosen to measure the particle size, and the morphology study was conducted by Phenom Pro Desktop SEMS-3400 scanning electron microscope (Thermo Fisher Scientific Inc., Waltham, USA). The particle size was calculated through Mastersizer 3000 from Malvern Panalytical (Worcestershire, United Kingdom). The percentage of production yield (PY) was calculated as equation listed below:

$$
P Y(\%)=\left(W_{S K @ M S}\right) /\left(W_{S P R C}+W_{K E T O}+W_{P L G A}\right)
$$

where $\mathrm{W}$ : the weight of corresponding component.

The measurement of loading efficiency and encapsulation efficiency were calculated through HPLC. Briefly, for the measurement of SPRC, ${ }^{48,49} 50$ mg of SK@MS was firstly dissolved in $2 \mathrm{~mL}$ of DCM with further extract SPRC with $10 \mathrm{~mL}$ distilled water and analysed with HPLC. As for the detection of KETO, $50 \mathrm{mg}$ of microspheres were dissolved in $2 \mathrm{~mL}$ of DCM and further diluted to a final volume of $10 \mathrm{~mL}$ through methanol. The solution was collected and analysed for the KETO concentration through HPLC as reported with little modification. ${ }^{50-52}$ Finally, the LE and EE were calculated as Equations (2) and (3) shown below:

$$
\begin{gathered}
L E(\%)=W_{E} / W_{S K @ M S} \\
E E(\%)=W_{E} / W_{T}
\end{gathered}
$$

where the LE: loading efficiency, EE: encapsulation efficiency, $\mathrm{W}_{\mathrm{E}}$ : weight of SPRC or KETO encapsulated in SK@MS, W $\mathrm{W}_{\mathrm{SK} @ \mathrm{Ms}}$ : weight of SK@MS, $\mathrm{W}_{\mathrm{T}}$ : weight of SPRC or KETO theoretically loaded in SK@MS.

\section{The Storage Stability Study of SK@MS}

The prepared SK@MS was placed in a vacuum desiccator under the condition of 4 or $25^{\circ} \mathrm{C}$ and $50 \%$ humidity to investigate storage environment effects. SK@MS was sampled at pre-determined intervals $(0,1,2,3,4,5,6$ month) to determine any changes in particle size as well as the loading efficiency. The SK@MS sampled at "0 month" were considered as control.

\section{In vitro Release Test}

The in vitro releasing experiment was conducted, using the method as reported with little modification. ${ }^{53}$ First, $50 \mathrm{mg}$ of differently prepared SK@MS was suspended in vials filled with $5 \mathrm{~mL}$ of PBS buffer $\left(37^{\circ} \mathrm{C}, \mathrm{pH}=7.4\right)$ with a constant shaking speed of $100 \mathrm{rpm}$ (Clifton Shaking Bath NE5, Nikel Electro Ltd., Weston-super-Mare, UK). At predetermined time intervals, $0.5 \mathrm{~mL}$ of the samples were taken out (equivalent amount of fresh PBS was used to refill) and centrifuged at 12,000 rpm for $5 \mathrm{~min}$. Samples were analyzed using a 1290 Infinity II LC System (Agilent Technologies Inc., Santa Clara, CA, USA).

\section{The Animal Ethics}

The Animal Care and Use Committee of Municipal Affairs Bureau of Macau approved all studies described herein (approval number AL010/DICV/SIS/2018), and experiment was conducted under the guidance of NIH Guide for the Care and Use of Laboratory Animals (8th edition). All experimental rats were housed in a $12 \mathrm{~h}$ light-dark cycle facility, with constant humidity and temperature, and a ventilation system was also equipped. The blood samples were withdrawn through the rat's tail vein, with a withdrawn volume of around $100 \mu \mathrm{L}$ per time. After experiment, the experimental rats were euthanized through carbon dioxide under the guidance of Macau University of Science and Technology (MUST) Guide for the Care and Use of Laboratory Animals.

\section{The Measurement of $\mathrm{H}_{2} \mathrm{~S}$ Level in vivo}

Blood samples were firstly dispersed in saline for subcutaneous injection, each sample contained the same amount of SPRC or KETO and were both calculated through the weight of the rats, which were $50 \mathrm{mg} \mathrm{kg}^{-1}$ for SPRC and $10 \mathrm{mg} \mathrm{kg}^{-1}$ for KETO. The rats' blood samples were collected at predetermined times $(0,1,2,6,12,24,36,48,60$, $72,84,96,108,120,132,144,156$, and 168 h) into heparin sodium tubes and analyzed. Each group contained 5 rats.

The concentration of $\mathrm{H}_{2} \mathrm{~S}$ was measured with the method reported with little modification, ${ }^{54,55}$ and the measurement scheme for detection of $\mathrm{H}_{2} \mathrm{~S}$ in vivo is shown in Figure 1. Briefly, $15 \mu \mathrm{L}$ of serum sample, $25 \mu \mathrm{L}$ of MBB 
A

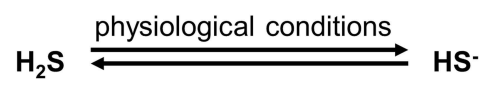

B

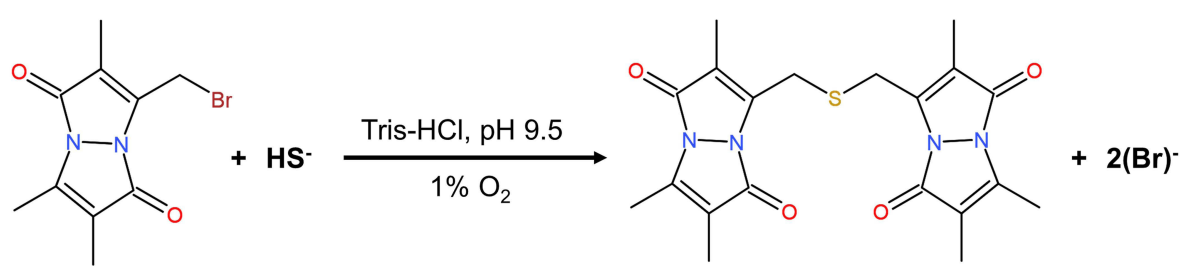

Figure I The mechanism of endogenous $\mathrm{H}_{2} \mathrm{~S}$ detection.

acetonitrile solution, and $35 \mu \mathrm{L}$ of $0.3 \%$ DTPA containing Tris-HCl buffer ( $\mathrm{pH}$ 9.5) were mixed and incubated in a hypoxia incubator for $30 \mathrm{~min}$. Subsequently, $25 \mu \mathrm{L}$ of sulfosalicylic acid was added to stop the reaction, and then centrifugated at $12,000 \mathrm{rpm}$ for $10 \mathrm{~min}$. Finally, $30 \mu \mathrm{L}$ of supernatant, $267 \mu \mathrm{L}$ of acetonitrile, and $3 \mu \mathrm{L}$ of internal standard (hydrocortisone methanol solution) were mixed and analyzed with LC-MS.

Samples were analyzed using an Agilent 1200 series HPLC system (Agilent Technologies Inc., Santa Clara, CA, USA) coupled with an Agilent 6460 Triple Quadrupole (Agilent Technologies Inc., Santa Clara, CA, USA). A ZORBAX Eclipse Plus $95 \mathrm{C} 18,2.1 * 50 \mathrm{~mm}, 1.8$ $\mu \mathrm{m}$ column (Agilent Technologies Inc., Santa Clara, CA, USA) was used and temperature was set at $35^{\circ} \mathrm{C}$. The mobile phase consisted of water (A) and acetonitrile (B) and the gradient delivery was as follows: at $0-0.5 \mathrm{~min}, 5 \%$ B; 0.5-0.6 min, 5-20\% B; 0.6-5.0 min, 20-47.5\% B; 5.0$5.1 \mathrm{~min}, 47.5-95 \% \mathrm{~B} ; 5.1-6.0 \mathrm{~min}, 95 \% \mathrm{~B}$, at a flow rate of $0.3 \mathrm{~mL} \mathrm{~min}$. The mass spectrometer was operated in positive ion mode. The scan type chosen was MRM with gas temperature at $325^{\circ} \mathrm{C}$ and gas flow at $10 \mathrm{~L} \mathrm{~min}^{-1}$. Scan time was $500 \mathrm{~ms}$ and start-stop mass was 100 1000. The sample injection volume was $5 \mu \mathrm{L}$.

\section{The Establishment of AIA Model in Rats}

The adjuvant induced arthritis (AIA) model of rat was established through the subcutaneous injection of CFA at tail base according to the manufacturer's instruction. Twenty-five rats weighting 180 200 $\mathrm{g}$ were randomly divided into five groups as follows: Control group $(\mathrm{n}=5)$, no intervention; AIA model group $(\mathrm{n}=5)$, SPRC group $(\mathrm{n}=5)$, KETO group $(\mathrm{n}=5)$, and SK@MS group $(\mathrm{n}=5)$, and the control group was injected with $0.1 \mathrm{~mL}$ of saline.

\section{The Drug Treatment Towards AIA in Rats} According to our previous study, ${ }^{45}$ the dose of $50 \mathrm{mg}$ $\mathrm{kg}^{-1}$ of SPRC was finally selected in this study, and the dose of KETO was then chosen $10 \mathrm{mg} \mathrm{kg}^{-1}$ for further study. After injection of CFA, the SPRC received subcutaneous injection of $50 \mathrm{mg} \mathrm{kg}^{-1}$ of SPRC, $10 \mathrm{mg} \mathrm{kg}^{-1}$ of KETO, and SK@MS (equivalent to $50 \mathrm{mg} \mathrm{kg}^{-1}$ of SPRC and $10 \mathrm{mg} \mathrm{kg}^{-1}$ of KETO) according to rats' body weight. The administration interval was chosen as once $\mathrm{q} 72 \mathrm{~h}$, and AIA model group received subcutaneous injection of $3 \mathrm{~mL}$ of saline.

\section{The Inhibition of Paw Swelling}

The paw volume was measured by a Ugo Basile 7140 plethysmometer (Ugo Basile, Gemonio VA, Italy) was measured at the 0, 5th, 10th, 15th, 20th, 25th, and 30th day post the injection of CFA. Arthritis index was scored from 0 to 4 per limb, for $0=$ no sign of inflammation, $1 \sim 4=$ increasing degrees of inflammatory, with a maximum score of 16 per rat. Table 1 shows the arthritis index scoring system. ${ }^{56}$

\section{The Von Frey Test in AIA Rats}

Rats were placed in transparent rat cages with wire mesh at the bottom. Al least let the rats have free access in cages for $30 \mathrm{~min}$ to ensure the rats could acclimate the new environment. Von Frey filaments with different force of 0.6, 1, 2, 4, $6,8,10$, and $15 \mathrm{~g}$ were applied to detect the pain threshold through the up-down method. ${ }^{57}$ With the slow increase of intensity, the mechanical withdrawal threshold was defined when the rats suddenly contracted. The mechanical withdrawal threshold was measured and recorded at the 0,5 th, 10th, 15th, 20th, 25th, and 30th day post the injection of CFA when observing the positive reaction. 
Table I The Arthritis Scoring System

\begin{tabular}{|l|l|}
\hline Arthritis Score & Degree of Inflammation \\
\hline 0 & No erythema and swelling \\
\hline & Erythema and mild swelling confined to the tarsals or ankle joint \\
2 & Erythema and mild swelling extending from the ankle to the tarsals \\
3 & Erythema and moderate swelling extending from the ankle to metatarsal joints \\
4 & Erythema and severe swelling encompassing the ankle, foot, and digits; ankylosis of the limb might be present \\
\hline
\end{tabular}

\section{Micro-CT Analyses}

The hind limb of rats in different groups were scanned and reconstructed as previously reported ${ }^{58}$ via Siemens Inveon PET/CT (Siemens, Munich, Germany). The exposure time was set as $800 \mathrm{~ms}$, while the voltage and electric current were set as $70 \mathrm{kV}$ and $400 \mathrm{~mA}$, respectively.

\section{The ELISA Examination of Inflammation in AIA Rats}

At day 30, blood sample was collected from rats in each group, the pro-inflammatory cytokines (TNF- $\alpha$, IL- $1 \beta$ and IL-6) and anti-inflammatory cytokine (IL-10) levels in serum were measured using ELISA kits according to the manufacturer's instructions.

\section{The Safety Evaluation}

Major organs including heart, liver, spleen, lung, kidney, small intestine, large intestine, and stomach were dissected and fixed with $4 \%$ paraformaldehyde and further stained with H\&E.

\section{Statistical Analysis}

Statistical analyses of samples were performed using GraphPad Prism. Each experiment is performed at least three times, and the data are expressed as the mean $\pm \mathrm{SD}$. Statistical significance was determined using a one-way analysis of variance (ANOVA) test, unless otherwise stated, $\mathrm{p}<0.05$ was considered significant.

\section{Results}

\section{The Characterization of SK@MS}

The preparation illustration of SK@MS is shown in Figure 2A. The SEM and DLS were firstly used to observe the morphology of SK@MS as shown in Figure 2B and C. A spherical-like, smooth-surface, and mono-dispersed microparticle could be observed under SEM and DLS, which indicated a successfully preparation of SK@MS. The particle size, production yield, LE, and EE are shown in Table 2. Through the double emulsion method, a PY around 79.89\% could be obtained, and the SK@MS showed a particle size around $25.12 \mu \mathrm{m}$, and the LE for SPRC and KETO were $6.47 \%$ and $2.64 \%$, respectively, while the EE for SPRC and KETO were $36.27 \%$ and $68.28 \%$, respectively. As Figure 2D and E illustrated, the SK@MS showed an excellent storage stability under $4^{\circ} \mathrm{C}$ or $25^{\circ} \mathrm{C}$ and $50 \%$ humidity, which indicated that SK@MS was stable, and, neither SPRC nor KETO undergo degradation under the storage conditions. The results of the in vitro release were shown in Figure 2F. As reported, ${ }^{47}$ together with our previous experiment (data not shown), $200 \mathrm{mg}$ of KETO could be successfully loaded into PLGA microspheres. However, a final amount of KETO of $45 \mathrm{mg}$ was chosen in this study. The reason we lowered the KETO loading amount was because high amount of KETO might induce potential side effect in vivo. Further detail would be illustrated below.

\section{The Elevation of $\mathrm{H}_{2} \mathrm{~S}$ in vivo}

The SPRC was reported to be able to promote the $\mathrm{H}_{2} \mathrm{~S}$ release in vivo, while to the best of our knowledge, there is no report on KETO could elevate the $\mathrm{H}_{2} \mathrm{~S}$ release in vivo. As shown in Figure 3A, SPRC group showed an instant $\mathrm{H}_{2} \mathrm{~S}$ elevation and $\mathrm{H}_{2} \mathrm{~S}$ metabolize within $6 \mathrm{~h}$ after single injection of SPRC solution, while SK@MS could slowly elevate the $\mathrm{H}_{2}$ $\mathrm{S}$ concentration in vivo at least $24 \mathrm{~h}$ after injection. Meantime, when co-injected SPRC and KETO, there is no significant change of the real-time $\mathrm{H}_{2} \mathrm{~S}$ level in vivo when compared with solely injection of SPRC. However, after 24h, as Figure 3B shown, the $\mathrm{H}_{2} \mathrm{~S}$ concentration showed decreasing in SK@MS group, meanwhile, the SPRC nor the SPRC + KETO group no more produced $\mathrm{H}_{2} \mathrm{~S}$ in vivo. Interestingly, $108 \mathrm{~h}$ after injection of the supplementations, the $\mathrm{H}_{2} \mathrm{~S}$ levels in all groups remains stable, which indicated a complete metabolism of endogenous $\mathrm{H}_{2} \mathrm{~S}$ produced via the supplementations (Figure 3C). It was observed that a stable $\mathrm{H}_{2} \mathrm{~S}$ level in vivo, which was same as the control group, could be observed after 
A

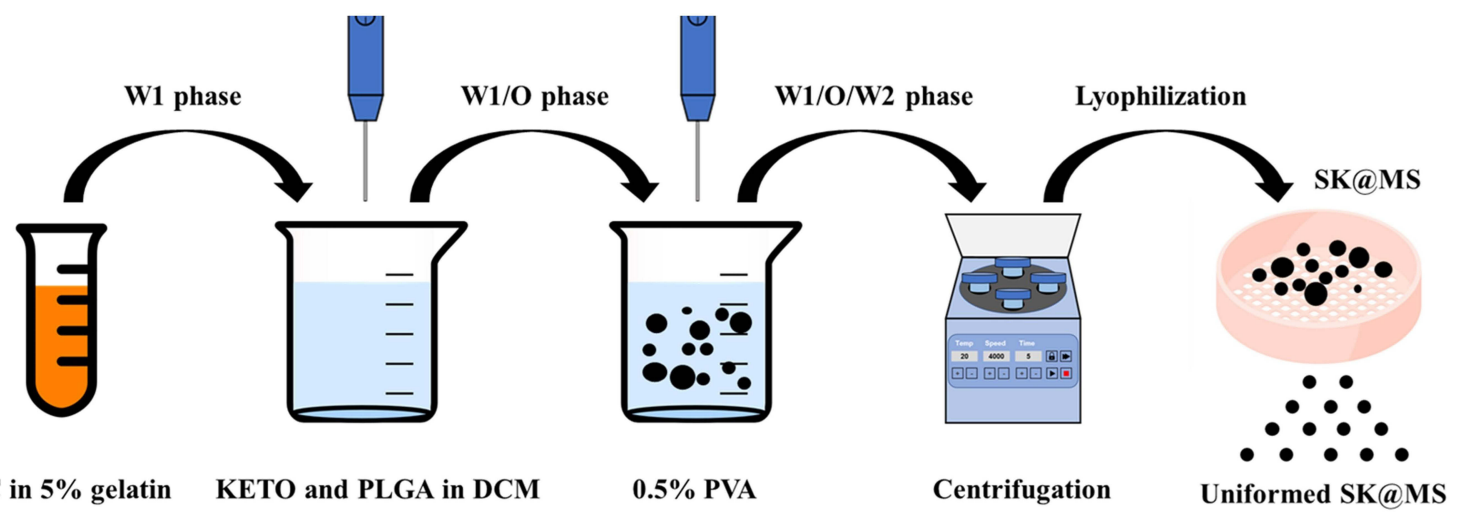

SPRC in $5 \%$ gelatin

B

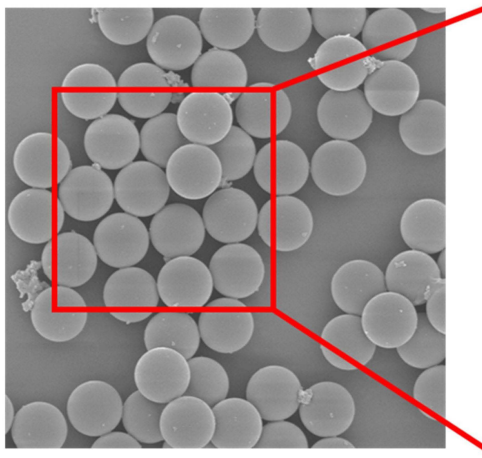

D

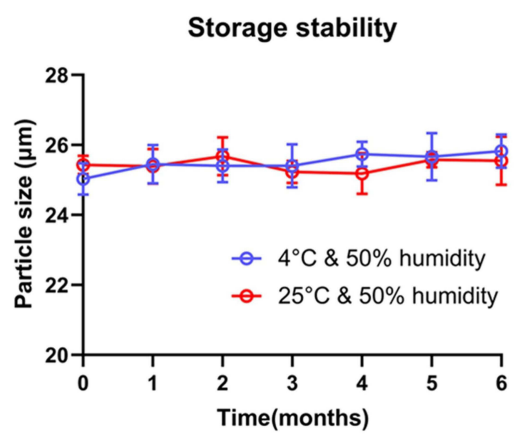

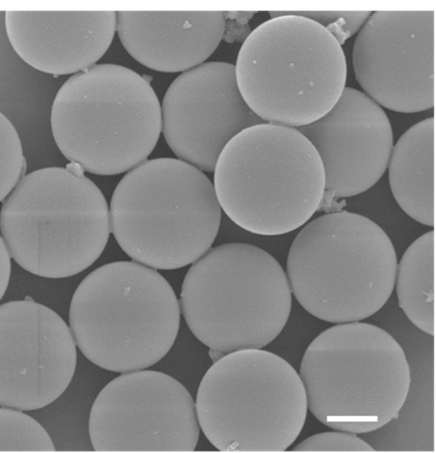

E

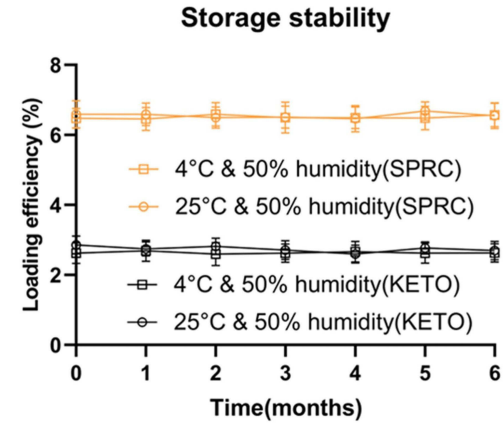

C

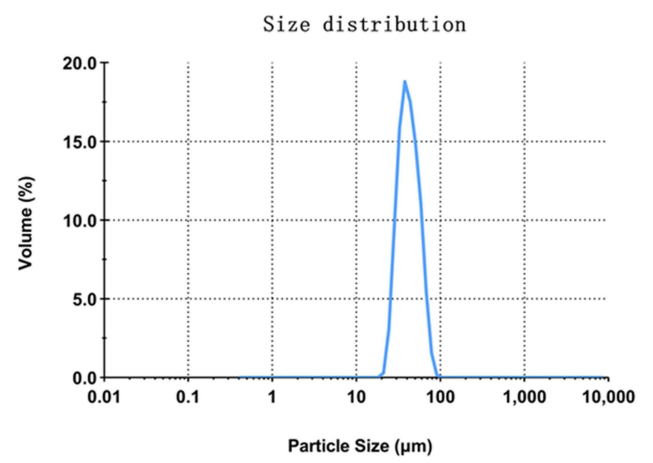

F

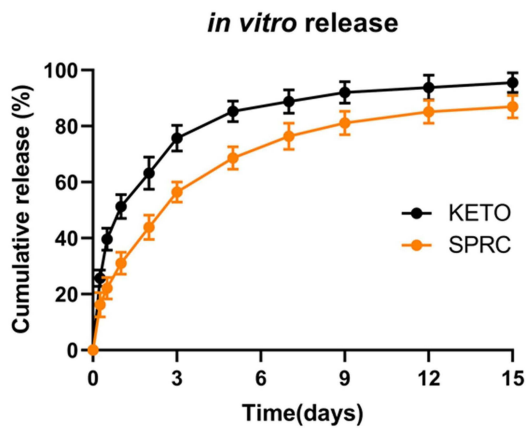

Figure 2 The preparation of SK@MS. (A) The schematic illustration of the preparation of SK@MS. (B) The SEM of SK@MS (scale bar=10 $\mu \mathrm{m}$ ). (C) The size distribution of SK@MS. (D and E) The SK@MS showed stable particle size and loading efficiency when stored under $4{ }^{\circ} \mathrm{C}$ or $25^{\circ} \mathrm{C}$ \& 50\% humidity, and (F) SK@MS showed a sustained release in vitro.

the administration of KETO, which indicated there is no promotion of endogenous $\mathrm{H}_{2} \mathrm{~S}$ when injected KETO solely (shown in Figure 3).

\section{The Anti-Inflammation Effect in AIA Rats}

In clinic, the RA patients usually have signs, such as swelling, erythema, and warmth. The anti-inflammation experiment design is illustrated in Figure 4A. As reported, the establishment of AIA usually accompanied with the body weight decrease in AIA model group, ${ }^{57}$ and this phenomenon could be observed in Figure 4B. The anti-edema effect of SK@MS towards AIA in rats was then evaluated through the measurement of paw volume and arthritis index. As shown in Figure 4C-D, no significant increase of neither paw volume nor arthritis index could be observed in the first 10 days. Starting from the 10th day, a dramatic increase of paw volume and arthritis index could be observed in AIA model group, and the SK@MS seems to suppress both the paw volume and arthritis index. Conversely, SPRC and KETO did not successfully inhibit the development of AIA progress, which indicated a synergistic effect of co-delivery of SPRC and KETO in the anti-edema. In Figure 4E, an erosion could be observed in 
Table 2 The Characterization Parameters of SK@MS ( $n=3$, Mean \pm SD)

\begin{tabular}{|l|c|}
\hline \multicolumn{2}{|l|}{ Characterization of SK@MS } \\
\hline Particle size / $\mu \mathrm{m}$ & $25.12 \pm 2.59$ \\
Production yield/\% & $79.89 \pm 5.84$ \\
LE (SPRC) $/ \%$ & $6.67 \pm 0.70$ \\
LE (KETO) /\% & $2.64 \pm 0.15$ \\
EE (SPRC) $/ \%$ & $37.20 \pm 2.96$ \\
EE (KETO) /\% & $68.28 \pm 3.73$ \\
\hline
\end{tabular}

Abbreviations: LE (SPRC) or LE (KETO), loading efficiency of either SPRC or KETO; EE (SPRC) or EE (KETO), encapsulation efficiency of either SPRC or KETO.

model group, and, in the SPRC and KETO group, which indicated low therapeutical effects of solely use of SPRC and KETO. However, the SK@MS group showed a dramatic therapeutical effect. The anti-inflammation effect of SK@MS was also evaluated by the measurement of plasma cytokines. As shown in Figure 4F-I, neither the SPRC group nor KETO group showed significant anti-inflammation effect in AIA rats compared with model group, while a dramatic decrease of pro-inflammatory cytokines (Figure $4 \mathrm{~F}-\mathrm{H}$ ) and increase of anti-inflammatory cytokine (Figure 4I) could be observed in SK@MS group.

\section{The Anti-Nociception Effect of SK@MS in AIA Rats}

The Von Frey test was taken for the investigation of the anti-nociception effect, while this experiment was also an important index to evaluate the severity of inflammation in

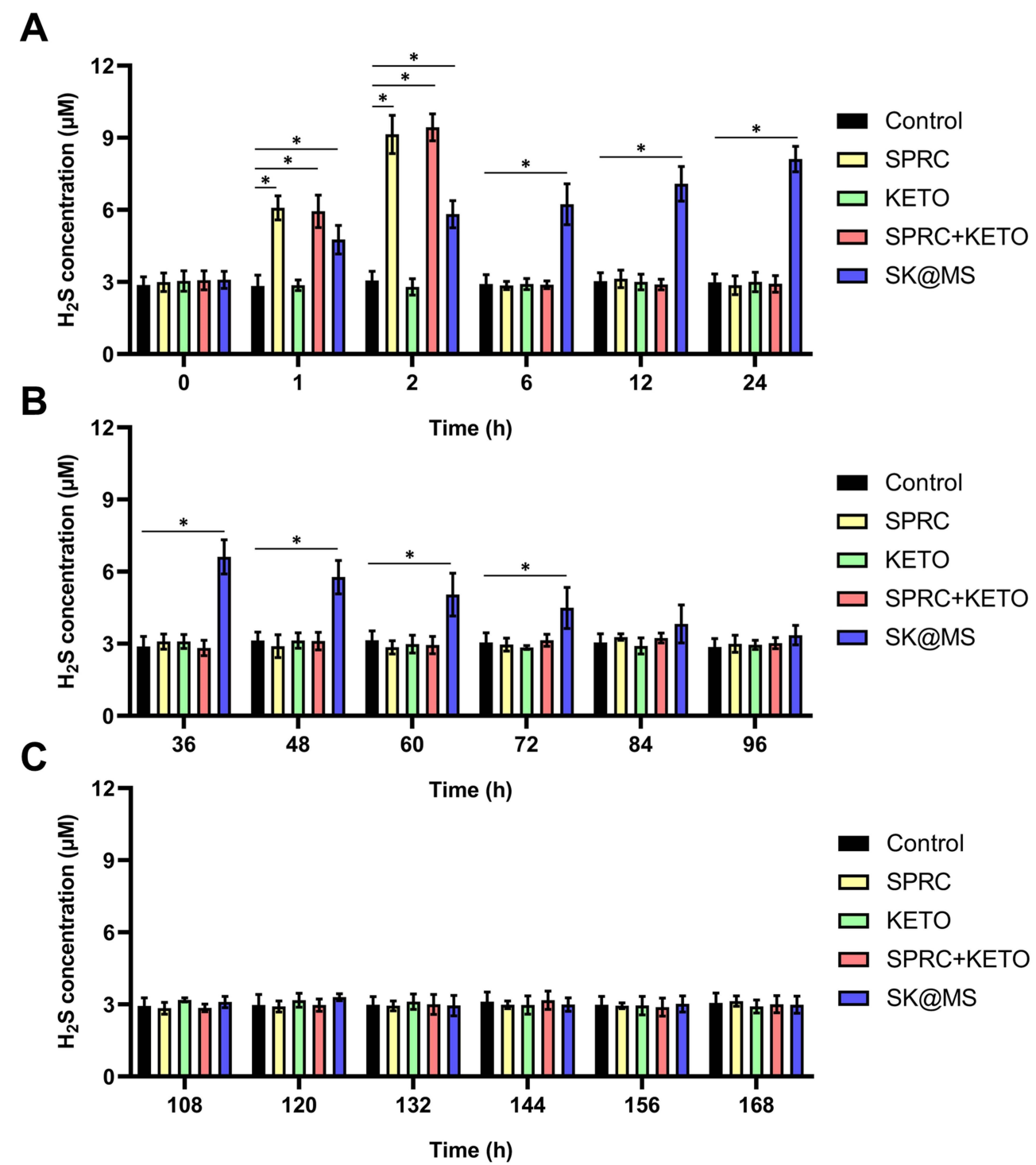

Figure 3 The real-time $\mathrm{H}_{2} \mathrm{~S}$ concentration in vivo after single injection of supplementations. (A) The first 24h post single injection, (B) the 36-96h post single injection, and (C) the 108-168h post single injection of supplementations. In this figure, *Indicated significant different compared with control group. ( $\mathrm{n}=5$, mean \pm SD). 
A

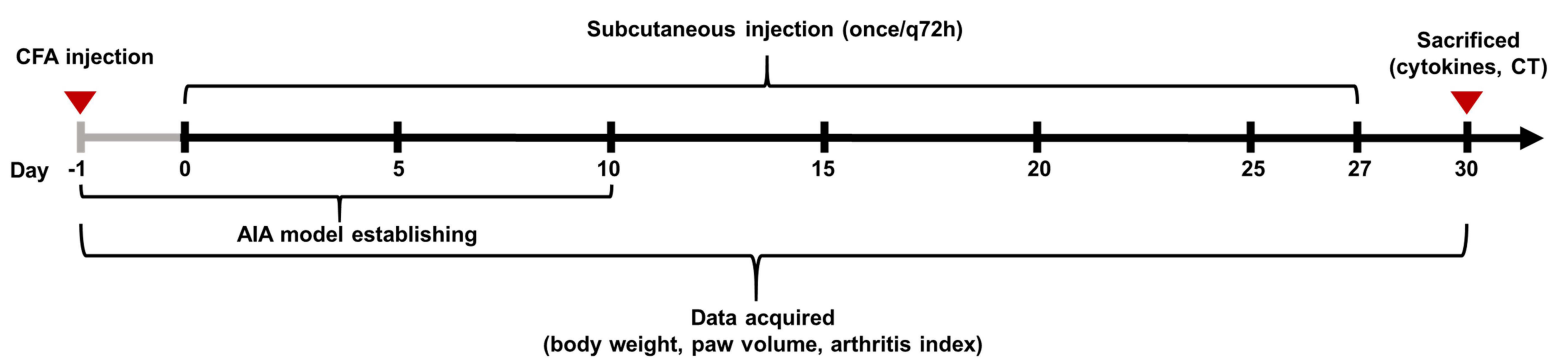

B

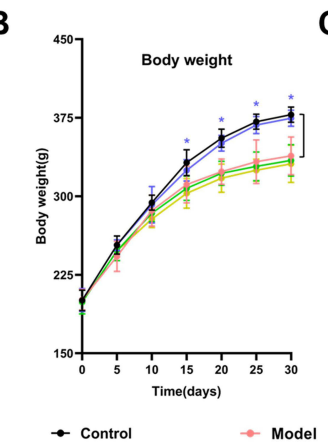

E

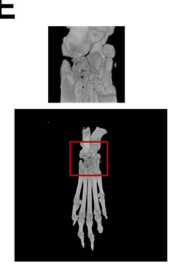

Control
C

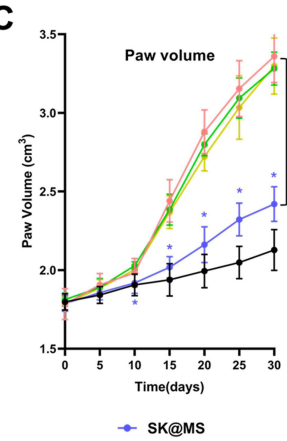

D

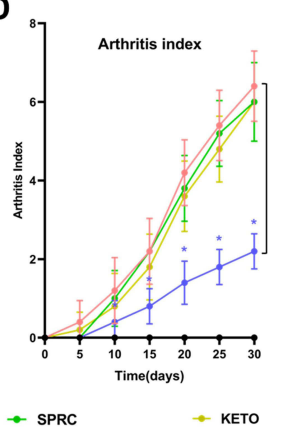

$F$

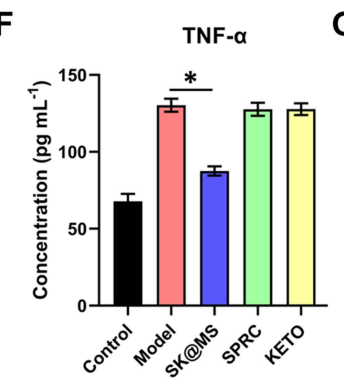

H

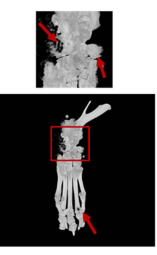

SPRC

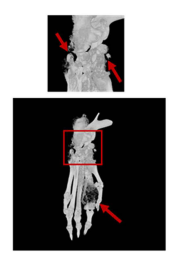

Model

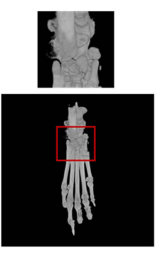

SK@MS

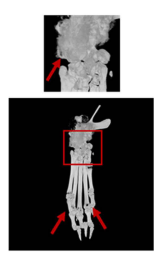

KETO

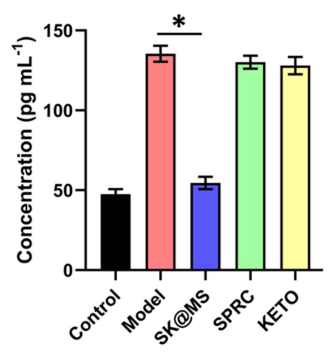

G IL-1 $\beta$
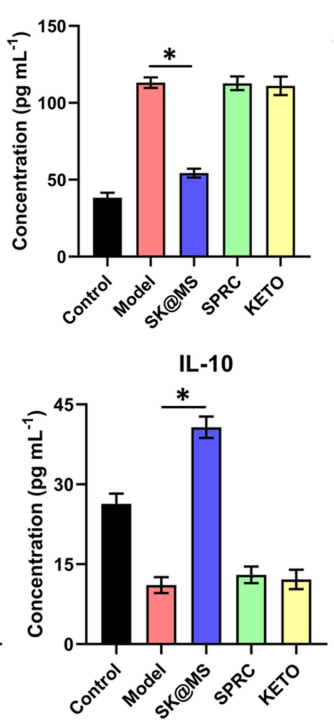

Figure 4 SK@MS could mitigate paw edema and inflammation in AIA rats. (A) Experiment design of AIA mitigation. (B) The body weight changes during the 30-day experimental period. (C) The paw volume and (D) arthritis index were measured to evaluate the severity of swollen symptoms. (E) Representative 3D reconstruction images of rats' paws from different treated groups. The pro-inflammatory cytokines levels of (F) TNF-a, (G) IL-Ib, and (H) IL-6 and anti-inflammatory cytokine level of (I) IL10 in rats were measured $(n=5$, mean $\pm S D)$. In this figure, *indicated significant different compared with model group.

AIA rats. The experiment design and timetable are shown in Figure 5A and B, respectively, while the results are shown in Figure 5C. Rats in control group showed a stable withdrawal threshold at $15 \mathrm{~g}$. Meantime, with the establishment of AIA, a dramatic decrease of withdrawal threshold could be observed. However, neither the SPRC group nor the KETO group could prevent the decreasing tendency of withdrawal threshold induced by AIA. Interestingly, the SK@MS group showed significant different withdrawal threshold when compared with the model group $(\mathrm{P}<0.05)$, which indicated the potential analgesic effect of SK@MS.

\section{The Safety Evaluation of SK@MS}

Chronic administration of high doses of SPRC generally leads to side effects such as organ injury, which might be caused via the persistent high $\mathrm{H}_{2} \mathrm{~S}$ concentration in vivo, and a long-term exposure to $\mathrm{H}_{2} \mathrm{~S}$ could harm organism. ${ }^{59,60}$ To evaluate the potential harm of supplementations to body, the heart, liver, spleen, kidneys, and lungs were resected from rats and examined by $\mathrm{H} \& \mathrm{E}$ staining. As shown in Figure 6A, compared with the control group, no tissue damage could be observed in any experimental groups, which revealed the low stimulation and toxicity of supplementations in vivo.

Besides, as a NSAIDs, the KETO might also showed gastrointestinal side effects in rats. ${ }^{61-63}$ Herein, the stomach, small intestine, and large intestine were also resected from rats and further examined through $\mathrm{H} \& \mathrm{E}$ staining. As shown in Figure 6B, no damage would be observed in any experimental groups, which revealed the low stimulation of supplementations to gastrointestinal system.

\section{Discussions}

PLGA is a pharmaceutical material approved by the FDA for its high biocompatibility and biodegradability. ${ }^{64}$ The PLGA microspheres formulations have drawn tremendous attention for it has abilities like sustained release, low toxicity, and 
A

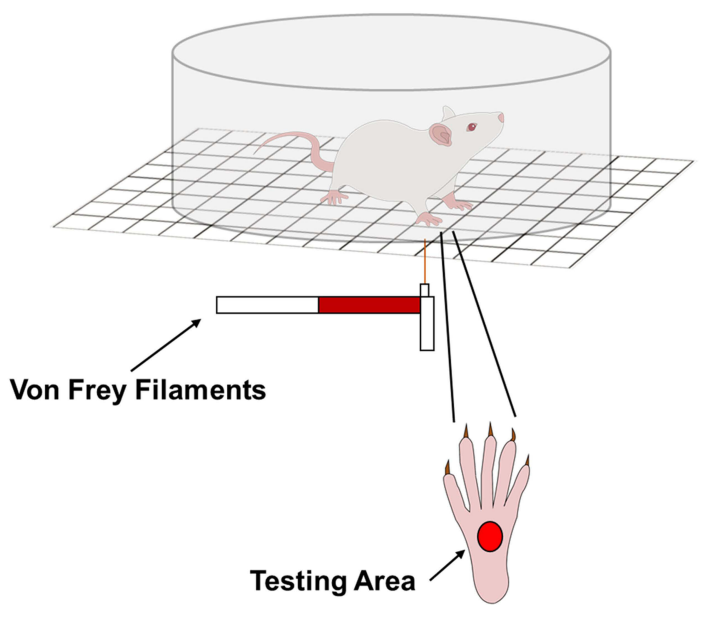

B

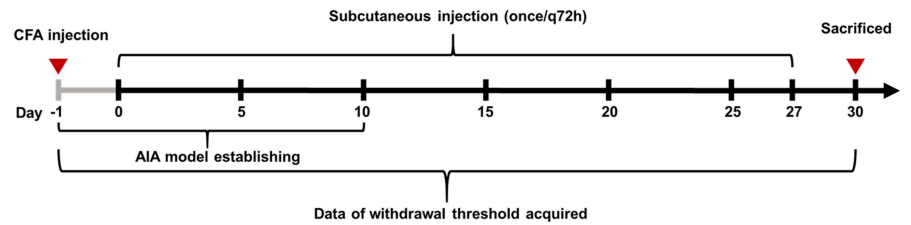

C

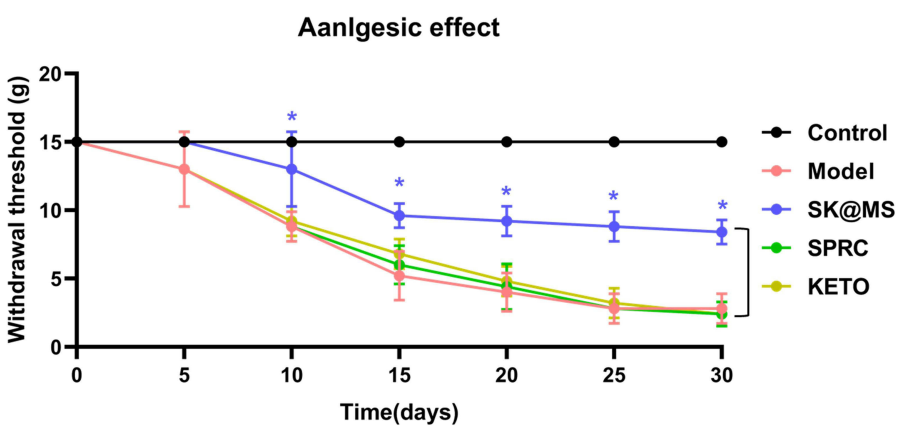

Figure $\mathbf{5}$ The anti-nociception experiment. (A) The experimental scheme illustration, and (B) the timetable of this experiment. (C) Changes in withdrawal threshold $\mathrm{s}$ in AIA rats of different treated groups $(n=5$, mean $\pm S D)$. In this figure, *indicated significant different compared with model group.

flattened blood concentration fluctuation. ${ }^{65-68}$ In this study, the releasing profile of KETO showed a two-stage releasing profile, which, were the initial burst releasing stage and sustained releasing stage. The KETO was encapsulated in the oil phase herein close to the particle surface, while the SPRC was in the inner water phase near the particle core, which explained the reason why the KETO appeared the initial burst release instead of the SPRC. Besides the initial burst release, a delayed SPRC could also be observed compared with the KETO, which further proved the distribution of SPRC near the particle core and KETO close to the particle surface.

Our previous study used SPRC with dosage of $100 \mathrm{mg} \mathrm{kg}^{-1}$ for the treatment of AIA in rats through subcutaneous injection or oral administration once $\mathrm{q} 72 \mathrm{~h}$, which showed gratifying therapeutical effect, while the dosage of $50 \mathrm{mg} \mathrm{kg}^{-1}$ showed limited therapeutical effect. ${ }^{55,58}$ However, in this study, we have different findings. In the SPRC group, we solely used SPRC at dosage of $50 \mathrm{mg} \mathrm{kg}^{-1}$, consistent with our previous studies, there was no treatment effects could be observed. When co-deliver SPRC and KETO in a PLGA microspheres (SK@MS) with a dosage equivalent to $50 \mathrm{mg} \mathrm{kg}^{-1}$ of SPRC, a dramatic therapeutical effect towards AIA could be observed. The reason for the improved therapeutical effect was possibly through two aspects, for one aspect, the sustained release of SPRC from SK@MS might elevate the $\mathrm{H}_{2} \mathrm{~S}$ concentration in vivo in a relevant stable level compared with SPRC powder. ${ }^{58,69}$ For another aspect, the co-deliver of NSAIDs might also augment the therapeutical potency of SPRC.
In the anti-nociception study, the rats in control group showed a stable withdraw threshold during the experimental period. However, with the establishment of AIA, a significant decrease in withdrawal threshold in AIA group could be observed, and this phenomenon was consistent with studies reported. $^{70,71}$ Theoretically, KETO, as an excellent analgesic drug, should show its anti-nociception effect when subcutaneous injected at the dosage of $2-5 \mathrm{mg} \mathrm{kg}^{-1} \mathrm{q} 24 \mathrm{~h}$ in rats. ${ }^{72,73}$ However, the limited anti-nociception effect was observed in this study even though we delivered the KETO at dosage of $10 \mathrm{mg} \mathrm{kg}^{-1}$, which was potentially due to the administration intervals was changed from $\mathrm{q} 24 \mathrm{~h}$ to $\mathrm{q} 72 \mathrm{~h}$. This result emphasized the importance of maintaining the KETO concentration in vivo.

In this study, all our experimental groups showed negligible damage to major organs in rats, while some reports indicated that therapeutic dose of KETO could cause severe gastrointestinal toxicity, such as acute gastrointestinal bleeding, erosions, and ulcers. ${ }^{72}$ The prolonged release manner of KETO (even higher dose than therapeutical dose) from SK@MS was possibly one reason for the decreased side effect, while the co-deliver of $\mathrm{H}_{2} \mathrm{~S}$ release donor might be another important reason.

\section{Conclusions}

In this study, the SPRC and KETO co-loaded PLGA microsphere (SK@MS) was successfully prepared, and the SK@MS showed a mono-dispersed spherical shape and sustained in vitro release (up-to 15 days). The 


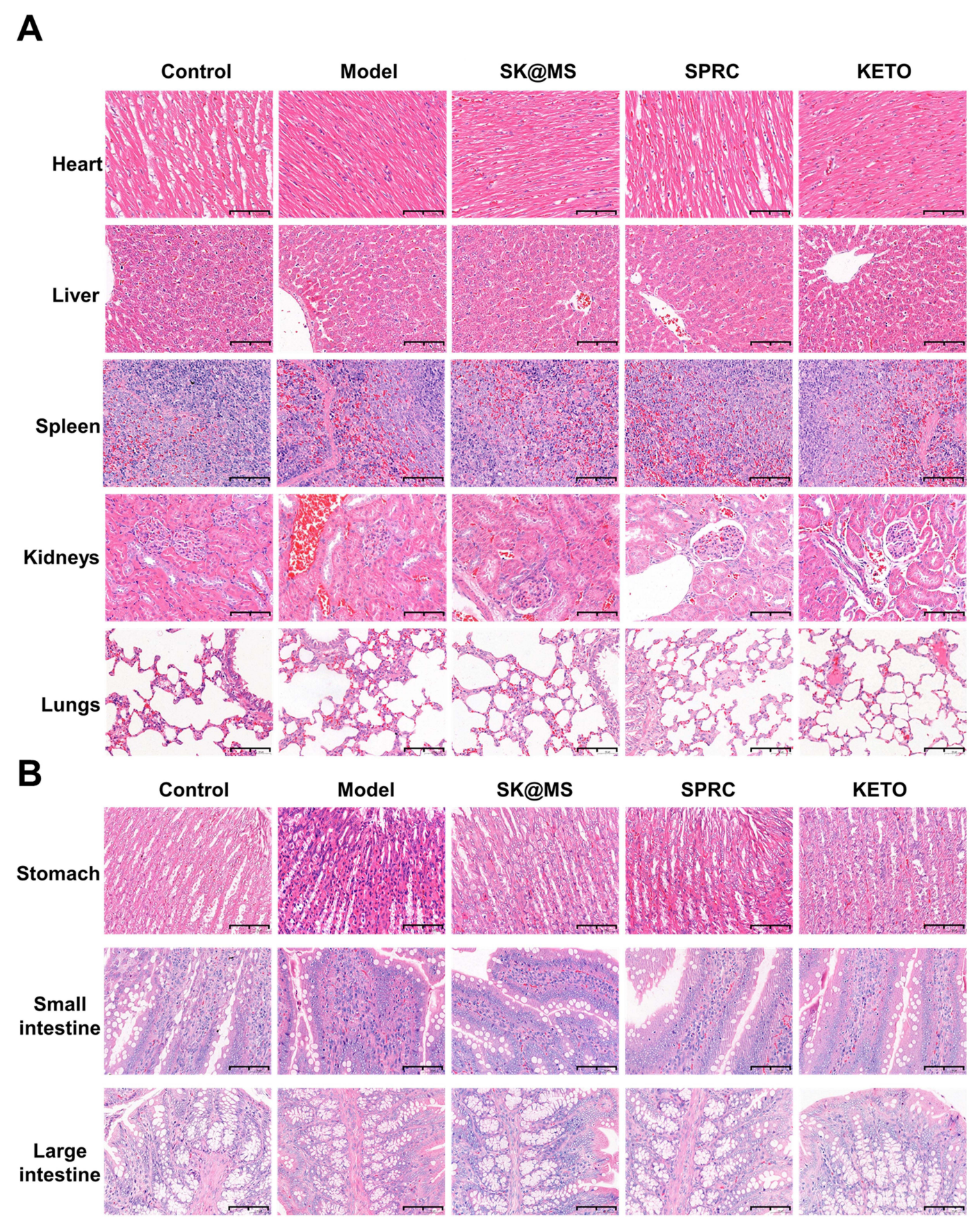

Figure 6 Evaluation of potential adverse effects of supplementations in experimental rats. H\&E staining was carried out for the examination of (A) heart, liver, spleen, kidneys, lungs, (B) and stomach, small intestine, large intestine after 30 days' experiment (scale bar $=100 \mu \mathrm{m}$ ).

SK@MS could sustained elevate the $\mathrm{H}_{2} \mathrm{~S}$ concentration in vivo, and what was more, the SK@MS also showed therapeutical effect (anti-inflammation and antinociception) against AIA in rats with low stimulation to major organs. Above all, it could be deduced that through co-delivery of $\mathrm{H}_{2} \mathrm{~S}$ donor and NSAIDs might promote the anti-inflammation effect of NSAIDs.

\section{Acknowledgments}

This work was supported by the Macau Science and Technology Development fund (FDCT) of (0067/2018/ A2, 033/2017/AMJ, 0007/2019/AKP, 0052/2020/A, 0011/ 2020/A1, 0030/2018/A1), and the National Natural Science Foundation of China (No. 81973320) to Yi Zhun Zhu. 


\section{Disclosure}

The authors report no conflicts of interest in this work.

\section{References}

1. Kokki H. Ketoprofen pharmacokinetics, efficacy, and tolerability in pediatric patients. Pediatr Drugs. 2010;12(5):313-329. doi:10.2165/ 11534910-000000000-00000

2. Mazìeres B. Topical ketoprofen patch. Drugs $R$ D. 2005;6 (6):337-344. doi:10.2165/00126839-200506060-00003

3. Cantisani C, Grieco T, Faina V, et al. Ketoprofen allergic reactions. Recent Pat Inflamm Allergy Drug Discov. 2010;4(1):58-64. doi:10.2174/187221310789895595

4. Boppana R, Kulkarni RV, Mohan GK, Mutalik S, Aminabhavi TM. In vitro and in vivo assessment of novel $\mathrm{PH}$-sensitive interpenetrating polymer networks of a graft copolymer for gastro-protective delivery of ketoprofen. RSC Adv. 2016;6(69):64344-64356. doi:10.1039/ C6RA04218J

5. Zeng Y, Lin X, Li F, et al. Ozonation of ketoprofen with nitrate in aquatic environments: kinetics, pathways, and toxicity. RSC Adv 2018;8(19):10541-10548. doi:10.1039/C7RA12894K

6. Kuczyńska J, Nieradko-Iwanicka B. Future prospects of ketoprofen in improving the safety of the gastric mucosa. Biomed Pharmacother. 2021;139:111608.

7. Gaskell H, Derry S, Wiffen PJ, Moore RA. Single dose oral ketoprofen or dexketoprofen for acute postoperative pain in adults. Cochrane Database Syst Rev. 2017;2017:CD007355.

8. Nie W, Xu P, Hao C, Chen Y, Yin Y, Wang L. Efficacy and safety of over-the-counter analgesics for primary dysmenorrhea a network meta-analysis. Medicine. 2020;99:e19881.

9. Derry S, Conaghan P, Da Silva JAP, Wiffen PJ, Moore RA. Topical NSAIDs for chronic musculoskeletal pain in adults. Cochrane Database Syst Rev. 2016;2016:CD007400.

10. Latosinski GS, Amzalak MJ, Pantoja JCF. Efficacy of ketoprofen for treatment of spontaneous, culture-negative, mild cases of clinical mastitis: a randomized, controlled superiority trial. J Dairy Sci. 2020;103(3):2624-2635. doi:10.3168/jds.2019-17504

11. Sintes GF, Bruckmaier RM, Wellnitz O. Nonsteroidal anti-inflammatory drugs affect the mammary epithelial barrier during inflammation. J Dairy Sci. 2020;103(11):10742-10753. doi:10.3168/ jds.2020-18818

12. Espinosa-Cano E, Aguilar MR, Portilla Y, Barber DF, Román JS. Anti-inflammatory polymeric nanoparticles based on ketoprofen and dexamethasone. Pharmaceutics. 2020;12(8):1-18. doi:10.3390/ pharmaceutics 12080723

13. Greig SL, Garnock-Jones KP. Loxoprofen: a review in pain and inflammation. Clin Drug Investig. 2016;36(9):771-781. doi:10.1007/s40261-016-0440-9

14. Mercadante S, Voza A, Serra S, et al. Analgesic efficacy, practicality and safety of inhaled methoxyflurane versus standard analgesic treatment for acute trauma Pain in the emergency setting: a randomised, open-label, active-controlled, multicentre trial in Italy (MEDITA). $A d v$ Ther. 2019;36(11):3030-3046. doi:10.1007/s12325-019-01055-9

15. Ong CKS, Seymour RA, Lirk P, Merry AF. Combining paracetamol (Acetaminophen) with nonsteroidal antiinflammatory drugs: a qualitative systematic review of analgesic efficacy for acute postoperative pain. Anesth Analg. 2010;110(4):1170-1179. doi:10.1213/ ANE.0b013e3181cf9281

16. Halen PK, Chagti KK, Giridhar R, Yadav MR. Combining anticholinergic and anti-inflammatory activities into a single moiety: a novel approach to reduce gastrointestinal toxicity of ibuprofen and ketoprofen. Chem Biol Drug Des. 2007;70(5):450-455. doi:10.1111/j.1747-0285.2007.00574.x
17. Cryer B, Feldman M. Cyclooxygenase-1 and cyclooxygenase-2 selectivity of widely used nonsteroidal anti-inflammatory drugs. $\mathrm{Am}$ J Med. 1998;104(5):413-421. doi:10.1016/S0002-9343(98)00091-6

18. Wu D, Wang H, Teng T, Duan S, Ji A, Li Y. Hydrogen sulfide and autophagy: a double edged sword. Pharmacol Res. 2018;131:120-127. doi:10.1016/j.phrs.2018.03.002

19. Olas B. Hydrogen sulfide in signaling pathways. Clin Chim Acta. 2015;439:212-218. doi:10.1016/j.cca.2014.10.037

20. Kumar M, Sandhir R. Hydrogen sulfide in physiological and pathological mechanisms in brain. CNS Neurol Disord Drug Targets. 2018;17(9):654-670. doi:10.2174/1871527317666180605072018

21. Wen YD, Wang H, Zhu YZ. The drug developments of hydrogen sulfide on cardiovascular disease. Oxid Med Cell Longev. 2018;2018:4010395.

22. Chen Z, Zhang M, Zhao Y. Hydrogen sulfide contributes to uterine quiescence through inhibition of NLRP3 inflammasome activation by suppressing the TLR4/NF-KB signalling pathway. $J$ Inflamm Res. 2021;14:2753-2768. doi:10.2147/JIR.S308558

23. Sidhapuriwala JN, Hegde A, Ang AD, Zhu YZ, Bhatia M. Effects of S-propargyl-cysteine (SPRC) in caerulein-induced acute pancreatitis in mice. PLoS One. 2012;7(3):e32574. doi:10.1371/journal. pone. 0032574

24. Wang Q, Wang XL, Liu HR, Rose P, Zhu YZ. Protective effects of cysteine analogues on acute myocardial ischemia: novel modulators of endogenous H2S production. Antioxid Redox Signal. 2010;12 (10):1155-1165. doi:10.1089/ars.2009.2947

25. Li W, Ma F, Zhang L, et al. S-propargyl-cysteine exerts a novel protective effect on methionine and choline deficient diet-induced fatty liver via Akt/Nrf2/HO-1 pathway. Oxid Med Cell Longev. 2016;2016:4690857.

26. Costa SKPF, Muscara MN, Allain T, et al. Enhanced analgesic effects and gastrointestinal safety of a novel, hydrogen sulfide-releasing anti-inflammatory drug (Atb-352): a role for endogenous cannabinoids. Antioxid Redox Signal. 2020;33(14):1003-1009. doi:10.1089/ars.2019.7884

27. Głowacka U, Magierowska K, Wójcik D, et al. Microbiome profile and molecular pathways alterations in gastrointestinal tract by hydrogen sulfide-releasing nonsteroidal anti-inflammatory drug (ATB-352): insight into possible safer polypharmacy. Antioxid Redox Signal. 2021. doi:10.1089/ars.2020.8240

28. Gugliandolo E, Fusco R, D'Amico R. Anti-inflammatory effect of ATB-352, a H2S -releasing ketoprofen derivative, on lipopolysaccharide-induced periodontitis in rats. Pharmacol Res. 2018;132:220-231. doi:10.1016/j.phrs.2017.12.022

29. Chen L, Qian M, Zhang L, et al. Co-delivery of doxorubicin and ShRNA of beclin1 by folate receptor targeted pullulan-based multifunctional nanomicelles for combinational cancer therapy. RSC $A d v$. 2018;8(32):17710-17722. doi:10.1039/C8RA01679H

30. Suraphan N, Fan L, Liu B, Wu D. Co-delivery of chlorantraniliprole and avermectin with a polylactide microcapsule formulation. RSC Adv. 2020;10(43):25418-25425. doi:10.1039/D0RA03825C

31. Xu C, Tian H, Sun H, Jiao Z, Zhang Y, Chen X. A PH sensitive co-delivery system of SiRNA and doxorubicin for pulmonary administration to B16F10 metastatic lung cancer. RSC Adv. 2015;5 (125):103380-103385. doi:10.1039/C5RA21934E

32. Wang GH, Yang HK, Zhao Y, Zhang DW, Zhang LM, Lin JT. Codelivery of doxorubicin and P53 by biodegradable micellar carriers based on chitosan derivatives. RSC Adv. 2015;5(128):105901-105907. doi:10.1039/C5RA19050A

33. Godsey ME, Suryaprakash S, Leong KW. Materials innovation for co-delivery of diverse therapeutic cargos. RSC Adv. 2013;3 (47):24794-24811. doi:10.1039/c3ra43094d

34. Chen AM, Zhang M, Wei D, et al. Co-delivery of doxorubicin and Bcl-2 SiRNA by mesoporous silica nanoparticles enhances the efficacy of chemotherapy in multidrug-resistant cancer cells. Small. 2009;5(23):2673-2677. doi:10.1002/smll.200900621 
35. Liu S, Guo Y, Huang R, et al. Gene and doxorubicin co-delivery system for targeting therapy of glioma. Biomaterials. 2012;33 (19):4907-4916. doi:10.1016/j.biomaterials.2012.03.031

36. Creixell M, Peppas NA. Co-delivery of SiRNA and therapeutic agents using nanocarriers to overcome cancer resistance. Nano Today. 2012;7(4):367-379. doi:10.1016/j.nantod.2012.06.013

37. Wang $\mathrm{H}$, Zhao $\mathrm{Y}, \mathrm{Wu} \mathrm{Y}$, et al. Enhanced anti-tumor efficacy by co-delivery of doxorubicin and paclitaxel with amphiphilic methoxy PEG-PLGA copolymer nanoparticles. Biomaterials. 2011;32 (32):8281-8290. doi:10.1016/j.biomaterials.2011.07.032

38. Liu W, Xi G, Yang X, et al. Poly(lactide-co-glycolide) grafted hyaluronic acid-based electrospun fibrous hemostatic fragments as a sustainable anti-infection and immunoregulation material. $J$ Mater Chem B. 2019;7(32):4997-5010. doi:10.1039/C9TB00659A

39. Sherwani MA, Tufail S, Khan AA, Owais M. Dendrimer-PLGA based multifunctional immuno-nanocomposite mediated synchronous and tumor selective delivery of SiRNA and cisplatin: potential in treatment of hepatocellular carcinoma. RSC Adv. 2015;5 (49):39512-39531. doi:10.1039/C5RA03651H

40. Thi Hiep N, Lee B-T. Electro-spinning of PLGA/PCL blends for tissue engineering and their biocompatibility. J Mater Sci. 2010;21 (6):1969-1978.

41. Ji W, Yang F, Seyednejad H, et al. Biocompatibility and degradation characteristics of PLGA-based electrospun nanofibrous scaffolds with nanoapatite incorporation. Biomaterials. 2012;33(28):6604-6614. doi:10.1016/j.biomaterials.2012.06.018

42. Anderson JM, Shive MS. Biodegradation and biocompatibility of PLA and PLGA microspheres. Adv Drug Deliv Rev. 2012;64:72-82. doi:10.1016/j.addr.2012.09.004

43. Kapoor DN, Bhatia A, Kaur R, Sharma R, Kaur G, Dhawan S. PLGA: a unique polymer for drug delivery. Ther Deliv. 2015;6 (1):41-58. doi:10.4155/tde.14.91

44. Arranz-Romera A, Davis BM, Bravo-Osuna I, et al. Simultaneous co-delivery of neuroprotective drugs from multi-loaded PLGA microspheres for the treatment of glaucoma. $J$ Control Release. 2019;297:26-38. doi:10.1016/j.jconrel.2019.01.012

45. Wu WJ, Jia WW, Liu XH, et al. S-propargyl-cysteine attenuates inflammatory response in rheumatoid arthritis by modulating the Nrf2-ARE signaling pathway. Redox Biol. 2016;10:157-167. doi:10.1016/j.redox.2016.08.011

46. Liu C, Gu X, Zhu YZ. Synthesis and biological evaluation of novel leonurine-SPRC conjugate as cardioprotective agents. Bioorganic Med Chem Lett. 2010;20(23):6942-6946. doi:10.1016/j. bmcl.2010.09.135

47. Yu C, Zhang X, Sun X, et al. Ketoprofen and microRNA-124 co-loaded poly (Lactic-Co-Glycolic Acid) microspheres inhibit progression of adjuvant-induced arthritis in rats. Int J Pharm. 2018;552 (1-2):148-153. doi:10.1016/j.ijpharm.2018.09.063

48. Tran BH, Yu Y, Chang L, et al. A novel liposomal S-propargylcysteine: a sustained release of hydrogen sulfide reducing myocardial fibrosis via TGF-B1/Smad pathway. Int $J$ Nanomed. 2019;14:10061-10077. doi:10.2147/IJN.S216667

49. Ma G, Zhang L, Zhang P, et al. Physicochemical characteristics and gastrointestinal absorption behaviors of s-propargyl-cysteine, a potential new drug candidate for cardiovascular protection and antitumor treatment. Xenobiotica. 2015;45:322-334.

50. Dvořák J, Hájková R, Matysová L, Nováková L, Koupparis MA, Solich P. Simultaneous HPLC determination of ketoprofen and its degradation products in the presence of preservatives in pharmaceuticals. J Pharm Biomed Anal. 2004;36(3):625-629. doi:10.1016/j.jpba.2004.07.018

51. Rhee YS, Choi JG, Park ES, Chi SC. Transdermal delivery of ketoprofen using microemulsions. Int $J$ Pharm. 2001;228(12):161-170. doi:10.1016/S0378-5173(01)00827-4
52. Zakeri-Milani P, Barzegar-Jalali M, Tajerzadeh H, Azarmi Y, Valizadeh H. Simultaneous determination of naproxen, ketoprofen and phenol red in samples from rat intestinal permeability studies: HPLC method development and validation. J Pharm Biomed Anal. 2005;39(3-4):624-630. doi:10.1016/j.jpba.20 05.04.008

53. Juhász Á, Ungor D, Berta K, Seres L, Csapó E. Spreadsheet-based nonlinear analysis of in vitro release properties of a model drug from colloidal carriers. J Mol Liq. 2021;328:115405. doi:10.1016/j. molliq.2021.115405

54. Zhu YZ, Zhong JW, Ho P, et al. Hydrogen sulfide and its possible roles in myocardial ischemia in experimental rats. J Appl Physiol. 2007;102(1):261-268. doi:10.1152/japplphysiol.0 0096.2006

55. Yu Y, Wang Z, Ding Q, et al. The preparation of a novel poly(Lactic Acid)-based sustained $\mathrm{H} 2 \mathrm{~S}$ releasing microsphere for rheumatoid arthritis alleviation. Pharmaceutics. 2021;13(5):742. doi:10.3390/ pharmaceutics 13050742

56. Chuang $\mathrm{CH}$, Cheng YC, Lin SC, et al. Atractylodin suppresses dendritic cell maturation and ameliorates collagen-induced arthritis in a mouse model. J Agric Food Chem. 2019;67(24):6773-6784. doi:10.1021/acs.jafc.9b01163

57. Deuis JR, Dvorakova LS, Vetter I. Methods used to evaluate pain behaviors in rodents. Front Mol Neurosci. 2017;10:284. doi:10.3389/ fnmol.2017.00284

58. Yu Y, Wang Z, Yang Q, et al. A novel dendritic mesoporous silica based sustained hydrogen sulfide donor for the alleviation of adjuvant-induced inflammation in rats. Drug Deliv. 2021;28 (1):1031-1042. doi:10.1080/10717544.2021.1921075

59. Zheng YT, Zhu JH, Ma G, et al. Preclinical assessment of the distribution, metabolism, and excretion of S-propargyl-cysteine, a novel H 2S donor, in Sprague-Dawley rats. Acta Pharmacol Sin. 2012;33(6):839-844. doi:10.1038/aps.2012.15

60. Saeedi A, Najibi A, Mohammadi-Bardbori A. Effects of long-term exposure to hydrogen sulfide on human red blood cells. Int J Occup Environ Med. 2015;6(1):20-25. doi:10.15171/ijoem.2015.482

61. Kantor TG. Ketoprofen: a review of its pharmacologic and clinical properties. Pharmacother J Hum Pharmacol Drug Ther. 1986;6 (3):93-102. doi:10.1002/j.1875-9114.1986.tb03459.x

62. Na M, Yiyun C, Tongwen X, et al. Dendrimers as potential drug carriers. part ii. prolonged delivery of ketoprofen by in vitro and in vivo studies. Eur J Med Chem. 2006;41(5):670-674. doi:10.1016/j. ejmech.2006.01.001

63. Lazzaroni M, Bianchi Porro G. Gastrointestinal side-effects of traditional non-steroidal anti-inflammatory drugs and new formulations. In: Proceedings of the Alimentary Pharmacology and Therapeutics. Blackwell Publishing Ltd. Vol. 20; 2004:48-58.

64. Lü J-M, Wang X, Marin-Muller C, et al. Current advances in research and clinical applications of PLGA-based nanotechnology. Expert Rev Mol Diagn. 2009;9(4):325. doi:10.1586/erm.09.15

65. Ma G. Microencapsulation of protein drugs for drug delivery: strategy, preparation, and applications. $J$ Control Release. 2014;193:324-340. doi:10.1016/j.jconrel.2014.09.003

66. Park K, Otte A, Sharifi F, et al. Formulation composition, manufacturing process, and characterization of poly(Lactide-Co-Glycolide) microparticles. $J$ Control Release. 2021;329:1150-1161. doi:10.1016/j.jconrel.2020.10.044

67. Andhariya JV, Choi S, Wang Y, Zou Y, Burgess DJ, Shen J. Accelerated in vitro release testing method for naltrexone loaded PLGA microspheres. Int $J$ Pharm. 2017;520(1-2):79-85. doi:10.1016/j.ijpharm.2017.01.050

68. Hua Y, Wang Z, Wang D, et al. Key factor study for generic longacting PLGA microspheres based on a reverse engineering of vivitrol ${ }^{\circledR}$. Molecules. 2021;26:1247. 
69. Yu Y, Wang Z, Ding Q, et al. The preparation of a novel poly(Lactic Acid)-based sustained H 2 S releasing microsphere for rheumatoid arthritis alleviation. Pharmaceutics. 2021;13:742.

70. Wang JF, Xu HJ, He ZL, Yin Q, Cheng W, Yi M. Crocin alleviates pain hyperalgesia in AIA rats by inhibiting the spinal Wnt5a/ $\beta$ catenin signaling pathway and glial activation. Neural Plast. 2020;2020. doi:10.1155/2020/4297483

71. Kang M, Jung I, Hur J, et al. The analgesic and anti-inflammatory effect of WIN-34B, a new herbal formula for osteoarthritis composed of Lonicera Japonica Thunb and Anemarrhena Asphodeloides BUNGE in vivo. $J$ Ethnopharmacol. 2010;131(2):485-496. doi:10.1016/j.jep.2010.07.025
72. A therapeutic dose of ketoprofen causes acute gastrointestinal B1. Ingenta Connect. Available from: https:/www.ingentaconnect.com/ content/aalas/jaalas/2012/00000051/00000006/art00012;jsessionid= 15oxmg6eue6rf.x-ic-live-01. Accessed September 19, 2021.

73. Perret-Gentil MI. Recommended surgical analgesic protocols for mice and rats; 2017

\section{Publish your work in this journal}

The Journal of Inflammation Research is an international, peerreviewed open-access journal that welcomes laboratory and clinical findings on the molecular basis, cell biology and pharmacology of inflammation including original research, reviews, symposium reports, hypothesis formation and commentaries on: acute/chronic inflammation; mediators of inflammation; cellular processes; molecular mechanisms; pharmacology and novel anti-inflammatory drugs; clinical conditions involving inflammation. The manuscript management system is completely online and includes a very quick and fair peerreview system. Visit http://www.dovepress.com/testimonials.php to read real quotes from published authors.

Submit your manuscript here: https://www.dovepress.com/journal-of-inflammation-research-journal 